American J. of Engineering and Applied Sciences 4 (3): 425-428, 2011

ISSN 1941-7020

(C) 2011 Science Publications

\title{
A Comparative Performance Analysis of Multiple-Input Multiple-Output using MATLAB with Zero Forcing and Minimum Mean Square Error Equalizers
}

\author{
Bara'u Gafai Najashi and Tan Xiaoheng \\ Center of Communication and Tracking, Telemetering and Command, \\ College of Communication Engineering, Chongqing University 400044, Chongqing PRC
}

\begin{abstract}
Problem statement: Multiple-Input Multiple-Output systems (MIMO) were regarded as one of the most promising technologies in field of wireless communication. Generally considered as one of the several forms of smart antenna technology, it offers considerable increase in data throughput and link range without additional bandwidth or transmit power. The general idea involves the use of several antennas at the transmitter and the receiver to improve system performance. One of the approaches employed in combating ISI in MIMO transmission was through the use of equalizers. Approach: In this study a proposed MIMO system was simulated using MATLAB software. The different equalization schemes Zero Forcing (ZF) equalizer and Minimum Mean Square Error (MMSE) which aid in the elimination of Inter Symbol Interference (ISI) thus improving overall performance were compared to analyze the BER of the designed system. Results: From the simulation results, the MMSE equalizer clearly had a better performance over the ZF equalizer in the region of about $3 \mathrm{~dB}$. Conclusion: MIMO transmission with MMSE equalization offers greater performance over ZF equalization. This helps in nullifying the effects of ISI thus improving overall performance. Thorough understanding of these techniques provides a good platform for future research in areas such as MIMO-OFDM.
\end{abstract}

Key words: Multiple-Input Multiple-Output (MIMO), Zero Forcing (ZF), designed system, Minimum Mean Square Error (MMSE), transmit antennas, multipath propagation, MIMO system, diversity gain, MIMO transmission

\section{INTRODUCTION}

One of the major bottlenecks encountered in the wireless channel has been multipath propagation. Over the years, methods have been devised to reduce its effect on the quality of information being transmitted. Multiple-Input Multiple-Output (MIMO) can be used to reduce power consumption and increase capacity it can also reduce both infrastructure deployment costs and combat shadow fading (Zhou et al., 2010).

Using multiple antennas both at the receiver and transmitter, Multiple Input Multiple Output systems are very famous for the high capacities and degrees of freedom that they provide in rich scattering environments (Ershadi and Bakhshi, 2010). MIMO has emerged as one of the most promising technologies in wireless communication due to its ability to turn multipath propagation normally considered as a drawback into a benefit for the user (Gesbert et al., 2003). In a MIMO system the data stream from an individual user is demultiplexed into $\mathrm{N}_{t}$ separate substreams where $\mathrm{N}_{t}$ is equal to the number of transmit antennas employed in such a system. The sub-streams are then encoded into channel symbols. It is common practice to implement the same data rates on all the transmitters but adaptive modulation could also be employed (Holter, 2001). The signals are then propagated through the wireless channel. The signals are received by $\mathrm{N}_{\mathrm{r}}$ receive antennas.

MIMO technology has much channel capacity by configuring many antennas on the transmitters and receivers compared to traditional SISO channel. Study proves that under the precondition of keeping the channel bandwidth and transmitting power fixed, system capacity is proportional to the smaller of the transmitting antennas number and the receiving antennas number. The other is the obvious spatial diversity. In view of the shortage of bandwidth spectrum nowadays, in order to transmit data with high speed and high capacity, such as in HDTV (High

Corresponding Author: Bara'u Gafai Najashi, Center of Communication and Tracking, Telemetering and Command, College of Communication Engineering, Chongqing University 400044, Chongqing PRC 
Am. J. Engg. \& Applied Sci., 4 (3): 425-428, 2011

Definition Television) we should have very high spectrum efficiency and the capacity of overcoming the channel fading in the environment of multi-path channel (Ling, 2010).

With this transmission scheme, there is a linear increase in spectral efficiency compared to a logarithmic increase in more traditional systems utilizing receive diversity or no diversity (Holter, 2001). The high spectral efficiencies attained by a MIMO system are enabled by the fact that in a rich scattering environment, the signals from each individual transmitter appear highly uncorrelated at each of the receive antennas. When the signals are conveyed through uncorrelated channels between the transmitter and receiver, the signals corresponding to each of the individual transmit antennas have attained different spatial signatures. The receiver can use these differences in spatial signature to simultaneously and at the same frequency separate the signals that originated from different transmit antennas (Holter, 2001).

Principle: Consider a MIMO system with two transmits antennas and two antennas at the receiver $(2 \times 2)$, the channel can be represented as Eq. 1 and 2:

$\mathrm{Y}=\mathrm{Hx}$

$H=\left(\begin{array}{ll}h_{11} & h_{12} \\ h_{21} & h_{22}\end{array}\right)$

where, $\mathrm{x}$ and $\mathrm{y}$ are $(2 \times 1)$ matrix representing transmitted and received signal on each antenna. The four entries of the channel matrix $\mathrm{H}$ correspond to the channel gains between the antennas as shown in Eq. 2 above. The given formulas 1 and 2 can be generalized to any number of transmit and receive antennas. MIMO broadly offers two advantages: Multiplexing gain and diversity gain (Duman and Ghrayeb, 2008).

Multiplexing gain: Multiple independent spatial channels are created in order to achieve multiplexing gain. Independent information sequences can be transmitted on each channel at the same time. The maximum number of channels is min $(M, N)$, where $M$ is the number of transmission antennas $(\mathrm{Tx})$ and $\mathrm{N}$ is the number of receive antennas $(\mathrm{Rx})$. As min $(\mathrm{M}, \mathrm{N})$ increases, the number of spatial channels increase linearly. The system capacity also increases linearly.

By Singular Value Decomposition (SVD), a matrix can be decomposed as follows Eq. 3:

$\mathrm{H}=\mathrm{USV}^{\mathrm{H}}$

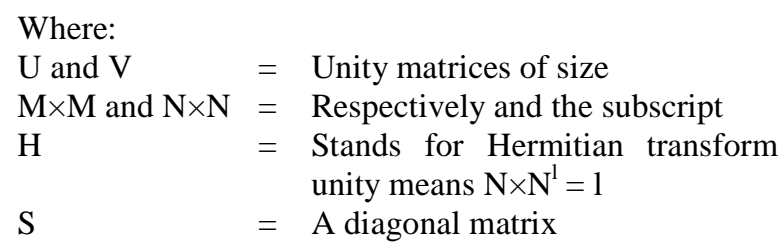

If we process the signal by multiplying $\mathrm{U}^{\mathrm{I}}$ at the transmit side and $\mathrm{V}$ at the receive side, then the equivalent channel will be like Eq. 4:

$$
\begin{aligned}
& \mathrm{U}^{\mathrm{l}}=\mathrm{U}^{\mathrm{H}} \mathrm{HV}=\left(\mathrm{U}^{\mathrm{H}} \mathrm{U}\right) \mathrm{S}\left(\mathrm{V}^{\mathrm{H}} \mathrm{V}\right) \\
& =\left(\begin{array}{cc}
\mathrm{S}_{11} & 0 \\
0 & \mathrm{~S}_{22}
\end{array}\right)
\end{aligned}
$$

$\mathrm{H}^{\mathrm{I}}$ is a diagonal matrix, with gains $\mathrm{S}_{11}$ and $\mathrm{S}_{22}$. Equation 1 then becomes Eq. 5:

$$
\left(\begin{array}{l}
y_{1} \\
y_{2}
\end{array}\right)=\left(\begin{array}{l}
S_{11} x_{1} \\
S_{22} x_{2}
\end{array}\right)
$$

this indicates that two independent channels are created (Penchong and Hong, 2009).

Diversity gain: Instead of two independent information sources, we can send some processed representation of a single information source in order to achieve diversity. The four channels can be seen as independent faded branches. So the MIMO channel now has a diversity order of $2 \times 2=4$ for generalized MIMO channel, the diversity order is $\mathrm{M} \times \mathrm{N}$.

For traditional systems like SISO, the problem of detection does not exist (Penchong and Hong, 2009). But for MIMO we have to separate the mixed signals at the receiver side. In the presence of noise, Eq. 1 becomes:

$\mathrm{Y}=\mathrm{Hx}+\mathrm{n}$

When the MIMO channel bandwidth is large relative to the channel's multipath delay spread, the channel suffers from ISI. One of the methods used in mitigating ISI in MIMO channels is by the use of equalizers. However, the equalizer is much more complex in MIMO channels since the channel must be equalized over both space and time (Goldsmith, 2005).

\section{MATERIALS AND METHODS}

System Model: A MIMO $2 \times 2$ transmission system consisting of 2 transmit antennas and two receive 
antennas was used. This configuration is probably the simplest form of MIMO transmission.

A multipath channel with a single tap was employed. The channel experienced by each transmit antenna is independent from the channel experienced by other transmit antennas. For the $\mathrm{i}^{\text {th }}$ transmit antenna to the $\mathrm{j}^{\text {th }}$ each transmit symbol gets multiplied by a randomly varying complex number $\mathrm{h}_{\mathrm{ji}}$. As the channel is a Rayleigh channel, the real and imaginary parts of $h_{i j}$ are Gaussian distributed having a mean and variance $\mu \mathrm{h}_{\mathrm{i}, \mathrm{j}}=0$ and variance $\sigma^{2} h_{j, i}=\frac{1}{2}$. Where by the channel experienced between each transmit to the receive antenna is independent and varying in time.

On the receive antenna, the noise $n$ has the Gaussian probability density function with $\mathrm{p}(\mathrm{n})=\frac{1}{\sqrt{2 \pi \sigma^{2}}} \mathrm{e}^{\frac{-(\mathrm{n}-\mu)^{2}}{2 \sigma^{2}}}$ with $\mu=0$ and $\sigma^{2}=\frac{\mathrm{N}_{0}}{2}$. It's assumed that the channel is known at the receiver

MIMO system design: The following steps were taken to design the system:

- Generate random binary sequence of +1 's and -1 's

- Modulate the binary sequence using Binary Phase Shift Keying (BPSK)

- Group them into pair of two and send two symbols in one time slot

- Pass them through a multipath fading channel with Additive White Gaussian Noise

- Perform equalization on the received signal

- Demodulate the BPSK symbols

- Calculate the bit error rate of the system

\section{RESULTS}

The effect of ZF equalizer and MMSE equalizer on MIMO transmission system is simulated in this study. The simulation results are given below.

Simulation results of MIMO with $\mathrm{ZF}$ equalizer: A random binary sequence is generated, which is then modulated using BPSK. The signal is then passed through a multipath fading channel with noise. At the receiver, equalization is performed using $\mathrm{ZF}$ equalizer. The BPSK symbols are demodulated and the BER is obtained by varying the value of the SNR as the Fig. 13 above indicates.

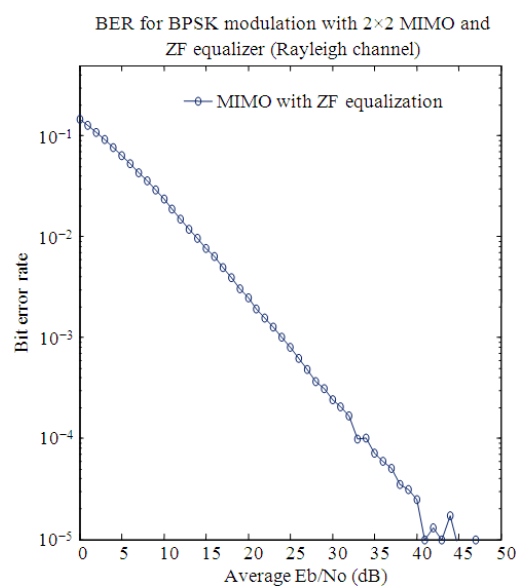

Fig. 1: BER for BPSK modulation with $2 \times 2 \mathrm{MIMO}$ and ZF equalizer

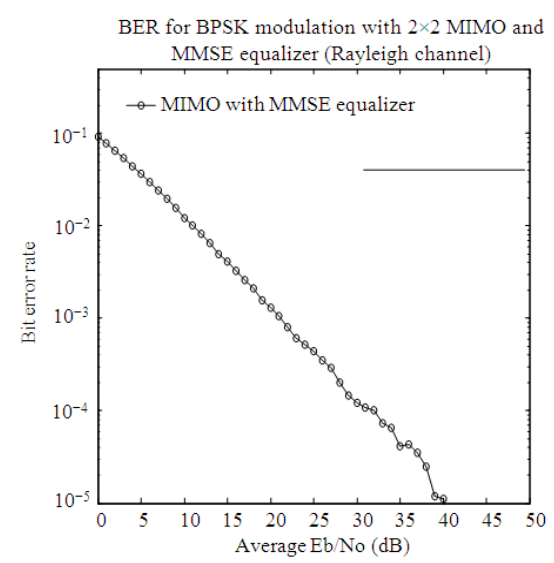

Fig. 2: BER for BPSK modulation with $2 \times 2 \mathrm{MIMO}$ and MMSE equalizer

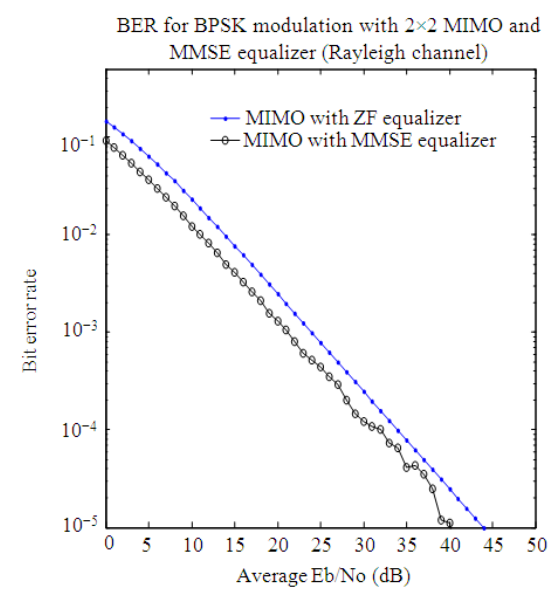

Fig. 3: Comparison of ZF and MMSE equalization with MIMO transmission 
Simulation results of MIMO with MMSE equalizer: An algorithm similar to the one employed above was employed to obtain the BER of the system with MMSE equalizer.

The comparison shows that MMSE equalizer has better performance of about $3 \mathrm{~dB}$ over $\mathrm{ZF}$ equalizer in a MIMO transmission.

\section{DISCUSSION}

In this study, the simulation of a MIMO transmission system was conducted due to its importance in modern wireless application. The problem of ISI due to multipath fading has been a bottleneck in digital communication systems. The demand for higher data rates and better quality of service cannot be achieved without mitigating the effect of ISI. MIMO technology is one of the techniques that can be applied to solve this problem as it turns multipath propagation from a disadvantage to an advantage. Furthermore, Zero Forcing and MMSE equalizers were simulated and the results compared. The results show that MMSE equalizer performs better than $\mathrm{ZF}$ equalizer.

\section{CONCLUSION}

In this simulation, the BER of a MIMO transmission with equalization was presented. The effect of Zero forcing Equalization and Minimum Mean Square Error Equalization using MATLAB were investigated. The simulation shows an improvement of about $3 \mathrm{~dB}$ in MMSE over ZF equalizer. This generally increases the performance of the overall system.

\section{REFERENCES}

Duman, T.M. and A. Ghrayeb, 2008. Coding for MIMO Communication Systems. 1st Edn., John Wiley and Sons, Chichester, England, ISBN: 0470028092, pp: 338.

Ershadi, E. and H. Bakhshi, 2010. A preamble-based frequency offset compensation scheme in multiple input multiple output orthogonal frequency division multiplexing systems. J. Comput. Sci., 6: 478-483. DOI: 10.3844 jessp.2010.478.483

Gesbert, D., M. Shafi, D.S. Shiu, P.J. Smith and A. Naguib, 2003. From theory to practice: An overview of MIMO space-time coded wireless systems. IEEE J. Select. Areas Commun., 21: 281-302. DOI: 10.1109/JSAC.2003.809458

Goldsmith, A., 2005. Wireless Communications. 1st Edn., Cambridge University Press, Cambridge, ISBN: 0521837162, pp: 644.

Holter, B., 2001. On the capacity of the MIMO channel-a tutorial introduction. Norwegian University of Science and Technology. http://new.iet.ntnu.no

Ling, W., 2010. MIMO simulation realization of DVB-T system based on MATLAB. Proceedings of the 3rd IEEE International Conference on Computer Science and Information Technology, Jul. 9-11, IEEE Xplore Press, Chengdu, pp: 387-390. DOI: 10.1109/ICCSIT.2010.5564146

Penchong, J. and Z. Hong, 2009. Development and simulation of a MIMO communication system based on IEEE $802.11 \mathrm{n}$. University of Science and Technology. http://people.ee.duke.edu

Zhou, X., D. Wang, D. Hu and Q. Lin, 2010. Performance study of a resource allocation scheme with fairness consideration in multihop systems over nakagami-m channels. Inform. Technol. J., 9: 266-273. 